\title{
A relevância da implementação de um programa de rastreamento de câncer de ovário: uma revisão narrativa
}

\author{
The relevance of implementing an ovarian cancer screening program: a narrative review
}

La relevancia de implementar un programa de cribado de cáncer de ovario: una revisión narrativa

Gabriel Jancik Rey Rodrigues ${ }^{1 *}$, Ana Luísa Terra Lasmar ${ }^{1}$, Bárbara Ellen Souza Rezende ${ }^{1}$, Bruna Cristina Silva Martins ${ }^{1}$, Higor Gomes Mussi ${ }^{1}$, Laura Nacife Rabello ${ }^{1}$, Sâmara Marques Campagnani ${ }^{1}$, Karine Ferreira dos Santos ${ }^{1}$.

\section{RESUMO}

Objetivo: Descrever os métodos de rastreamento pesquisados para câncer de ovário, que são: Ultrassom Transvaginal (UTV), pesquisa do antígeno CA-125, e doppler e as suas limitações. Revisão bibliográfica: Cada método apresenta suas características positivas e negativas, que impedem sua implementação como método de rastreio. A proteína sérica $\mathrm{CA}-125$ é um ótimo marcador, porém gera vários resultados falsos positivos; o doppler, por outro lado, apresenta sensibilidade e especificidade razoáveis para o diagnóstico, mas não são capazes de detectar pequenas lesões, o que é essencial para o diagnóstico inicial; e, por fim, o ultrassom transvaginal tem um baixo custo, não é invasivo e é amplamente aceito, mas não consegue identificar tumores na camada epitelial do ovário. Considerações finais: Apresenta informações acerca dos métodos de rastreamento discutidos atualmente e suas limitações, que impedem que o rastreamento do câncer de ovário seja implementado nos dias atuais. Com a alta taxa de letalidade desta doença, há um esforço mundial para diagnosticar câncer de ovário em estágio inicial e consequentemente reduzir a mortalidade, para isso, estudos estão sendo cada vez mais produzidos.

Palavras-chave: Neoplasias ovarianas, Rastreamento de câncer, Ultrassonografia.

\begin{abstract}
Objective: To describe the researched screening methods for ovarian cancer, which are: transvaginal ultrasound, CA-125 antigen search, and doppler and their limitations. Bibligraphic review: each method has its positive and negative characteristics, which prevent its implementation as a screening method. Serum protein CA-125 is a great marker, but it generates many false positives; Doppler, on the other hand, has reasonable sensitivity and specificity for diagnosis, but is not capable of detecting small lesions, which is essential for initial diagnosis; and finally, transvaginal ultrasound is inexpensive, non-invasive, and widely accepted, but it cannot identify tumors in the epithelial layer of the ovary. Final considerations: This study is extremely important to disseminate information about the currently discussed screening methods and their limitations, which prevent ovarian cancer screening from being implemented nowadays. With the high lethality rate of this disease, there is a worldwide effort to diagnose ovarian cancer at an early stage and consequently reduce mortality, for this, studies are being increasingly produced.
\end{abstract}

Keywords: Ovarian neoplasms, Cancer screening, Ultrasonography.

\section{RESUMEN}

Objetivo: Describir los métodos de cribado investigados para el cáncer de ovario, que son: ecografía transvaginal, búsqueda de antígeno CA-125 y doppler y sus limitaciones. Revisión bibliográfica: Cada método tiene sus características positivas y negativas, que impiden su implementación como método de cribado. La proteína sérica CA-125 es un gran marcador, pero genera muchos falsos positivos; El Doppler, por otro lado, tiene una sensibilidad y especificidad razonables para el diagnóstico, pero no es capaz de detectar lesiones pequeñas, lo cual es esencial para el diagnóstico inicial; y finalmente, la ecografía

${ }^{1}$ Pontifícia Universidade Católica de Minas Gerais (PUC Minas), Betim - MG.

*E-mail: gabrieljancik@gmail.com

SUBMETIDO EM: 7/2021

ACEITO EM: 7/2021

PUBLICADO EM: 9/2021 
transvaginal es económica, no invasiva y ampliamente aceptada, pero no puede identificar tumores en la capa epitelial del ovario. Consideraciones finales: Este estudio es de suma importancia para difundir información sobre los métodos de cribado actualmente discutidos y sus limitaciones, que impiden que se implementen en la actualidad los cribados del cáncer de ovario. Con la alta tasa de letalidad de esta enfermedad, existe un esfuerzo mundial por diagnosticar el cáncer de ovario en una etapa temprana y consecuentemente reducir la mortalidad, para ello, cada vez se realizan más estudios.

Palabras clave: Neoplasias de ovario, Cribado del câncer, Ecografia.

\section{INTRODUÇÃO}

Os ovários são as glândulas reprodutivas do sistema genital feminino. Estão localizados na pelve, um de cada lado do útero, e são responsáveis pela produção dos gametas femininos, ou óvulos, ao final da puberdade. Produzem também hormônios como estrogênio e progesterona, que controlam o desenvolvimento dos caracteres sexuais secundários e atuam sobre o útero na implantação do óvulo fecundado e no início do desenvolvimento do embrião. Na velhice, os ovários diminuem de tamanho e, por volta dos 50 anos, eles não produzem mais os óvulos (DANGELO J e FATTINI C, 2007).

Entre as neoplasias malignas que acometem a mulher, o câncer de ovário é o quinto mais comum, e, dentre os cânceres ginecológicos, é o segundo mais frequente e o mais letal, o que é evidenciado pela taxa de sobrevida em 5 anos de apenas $46 \%$. Anualmente no mundo, são identificados 250.000 novos casos e registrados 140.000 óbitos. No Brasil, foi estimado um total de 6.650 diagnósticos para 2020 e foram registradas 4.123 mortes em 2019. Dentre os quadros de neoplasias ovarianas, 95\% são provenientes das células epiteliais e o restante tem origem em células germinativas, que formam os óvulos, e em células estromais, que produzem a maior parte dos hormônios femininos (INSTITUTO NACIONAL DE CÂNCER, 2021; KAMAL R, et al., 2018; CAMPBELL S e GENTRY-MAHARAJ A, 2018).

O risco de câncer de ovário ao longo da vida é de aproximadamente 1,3\% na população geral (NEBGEN DR, et al., 2020). Ademais, dentro da população feminina esse câncer afeta 1 a cada 2.500 mulheres na pós-menopausa, quando essa doença se torna mais importante, e somente $9 \%$ dos casos ocorrem em mulheres com menos de 50 anos (CAMPBELL S e GENTRY-MAHARAJ A, 2018; MORERA AC, et al., 2020).

Dentre os principais fatores que aumentam as chances de desenvolver o câncer ovariano, é possível citar o excesso de peso corporal e o aumento da idade, sendo a idade média de apresentação da doença entre 60 a 63 anos. O histórico familiar de cânceres de ovário e de mama, que se configura como história positiva em parentes de primeiro ou segundo grau, também indicam uma predisposição ao desenvolvimento de neoplasia ovariana. Fatores como menarca precoce, que corresponde à primeira menstruação com menos de 12 anos, e menopausa tardia, que é caracterizada pela interrupção da menstruação após os 52 anos, também podem ter associação com um risco aumentado de câncer de ovário, porque aumentam o tempo de exposição ao estrogênio (INSTITUTO NACIONAL DE CÂNCER, 2021; KAMAL R, et al., 2018).

É imprescindível mencionar como fatores de risco os fatores genéticos, que correspondem às mutações nos genes BRCA1 e BRCA2, capazes de aumentar as chances de desenvolvimento do câncer de ovário ao longo da vida em $46 \%$ e $17 \%$, respectivamente. Além dessas mutações é possível citar também a mutação no gene supressor de tumor, o TP53. A alteração desse gene está associada principalmente ao carcinoma de ovário seroso de alto grau, que está presente em até $80 \%$ dos casos. Vale ressaltar que, em decorrência da mutação do Gene TP53, o indivíduo portador da alteração perde o principal produtor da P53, responsável por regular o ciclo celular, reparar o DNA da célula e, quando necessário, induzir a apoptose celular (CAMPBELL S e GENTRY-MAHARAJ A, 2018; NEBGEN DR, et al., 2020; FORSTNER R, 2020).

Em contrapartida, existem alguns fatores não genéticos que conferem proteção contra o câncer ovariano, diminuindo a chance de desenvolvê-lo, como a gravidez, a maior duração da amamentação, o uso prolongado de pílulas anticoncepcionais orais e a laqueadura tubária bilateral, que diminui o risco em aproximadamente 50\% (NEBGEN DR, et al., 2020). 
Apesar do diagnóstico precoce do câncer de ovário oferecer diversos benefícios, não há evidência científica de que seu rastreamento traga mais benefícios que riscos, e por isso este ainda não é oferecido (INSTITUTO NACIONAL DE CÂNCER, 2021).

Em contrapartida, esse artigo de revisão evidenciou que o diagnóstico precoce do câncer de ovário contribuiria para a diminuição da mortalidade na população feminina, e por isso esse estudo tem o objetivo de descrever os métodos de rastreamento já pesquisados para câncer de ovário, como Ultrassom Transvaginal (UTV), pesquisa do antígeno CA-125 e doppler, as taxas de sucesso dos mesmos e o motivo pelo qual tais métodos ainda não são utilizados como métodos de rastreamento.

\section{REVISÃO BIBILIOGRÁFICA}

Os cânceres de ovário podem ser divididos em carcinoma tipo I, que são, geralmente, neoplasias indolentes e de crescimento lento; e em carcinoma tipo II, que corresponde a neoplasias clinicamente agressivas e que podem se desenvolver de novo a partir da tuba e/ou do epitélio da superfície ovariana (KOSHIYAMA M, et al., 2017; CAMPBELL S e GENTRY-MAHARAJ A, 2018; NEBGEN DR, et al., 2020).

O carcinoma tipo I inclui o carcinoma endometrióide, o carcinoma de células claras e o carcinoma seroso de baixo grau, que é mais raro e caracterizado por um baixo número de mutações genéticas. $O$ carcinoma tipo II inclui o carcinoma seroso de alto grau, que é responsável por $68 \%$ a $75 \%$ dos cânceres de ovário, têm o pior prognóstico e representa $90 \%$ das mortes pela doença, pois corresponde a neoplasias clinicamente agressivas de alto grau e de crescimento rápido, que geralmente são diagnosticadas em estágio avançado, já que é difícil sua detecção em estágios iniciais. Este tipo de câncer geralmente apresenta uma mutação no gene TP53 (KOSHIYAMA M, et al., 2017; CAMPBELL S e GENTRY-MAHARAJ A, 2018; NEBGEN DR, et al., 2020).

Alguns autores acreditam que a maioria dos cânceres do tipo II (carcinoma seroso de ovário) tem como origem o carcinoma seroso intraepitelial da tuba de Falópio, por serem morfologicamente e molecularmente semelhantes. Nesse caso, acredita-se que as células da parte distal da trompa se implantam no ovário e viram um precursor para a doença. Diante disso, alguns estudos direcionaram estratégias futuras para a detecção em um estágio pré-maligno, porém nenhum rastreamento é capaz de detectar o carcinoma seroso intraepitelial da tuba de Falópio (CAMPBELL S e GENTRY-MAHARAJ A, 2018; NEBGEN DR, et al., 2020).

Infelizmente, $60 \%$ dos casos de câncer de ovário são diagnosticados de forma tardia, principalmente em estágios III (51\% dos casos), que corresponde à disseminação do câncer por toda a cavidade peritoneal, e IV (29\% dos casos). Tal fato determina um prognóstico ruim em consequência da grande extensão e do difícil tratamento, o que aumenta a mortalidade em decorrência dessa patologia, e por isso as taxas de sobrevida em 5 anos nesses estágios são inferiores a 30\%. O câncer de ovário raramente é diagnosticado em fase inicial, já que apresenta poucos sintomas e estes, quando presentes, são inespecíficos (CAMPBELL S e GENTRY-MAHARAJ A, 2018; NEBGEN DR, et al., 2020).

Em contrapartida, quando diagnosticado precocemente, as taxas de sobrevivência em 5 anos são de $90 \%$ se o câncer for confinado ao ovário, caracterizando o estágio I, ou de $70 \%$ se confinado à pelve, classificado como Estágio II (NEBGEN DR, et al., 2020).

Para que o método de rastreamento de um câncer seja viável e tenha sucesso, deve-se conhecer bem a história natural da patologia a ser rastreada, é necessária a existência de um tratamento eficaz que permita a cura da doença quando detectada em estágio inicial e o método deve ser barato, fácil de usar, rápido, indolor, pouco constrangedor e possuir alta especificidade e sensibilidade, de pelo menos 99,6\%, para que seja possível um valor preditivo positivo de no mínimo 10\% (KAMAL R, et al., 2018; CAMPBELL S e GENTRY-MAHARAJ A, 2018; MORERA AC, et al., 2020).

Em decorrência da alta taxa de mortalidade, sintomatologia inespecífica e grande chance de recidiva quando em estágio mais avançados há um grande esforço mundial para pesquisar métodos de triagem para detecção precoce da neoplasia de ovário, em estágios iniciais. Nesse contexto, estão sendo realizadas buscas por programas de rastreamento de cânceres, que têm como finalidade aumentar a expectativa de 
vida dos pacientes e reduzir as taxas de mortalidade em decorrência desta patologia, através do diagnóstico em estágios iniciais, para permitir uma maior possibilidade de cura (BUHLING KJ, et al., 2016; KAMAL R, et al., 2018; FORSTNER R, 2020).

No que tange o câncer de ovário, os métodos de rastreamento mais investigados são o ultrassom transvaginal UTV, que tem como objetivo avaliar a morfologia ovariana nos planos longitudinal e transversal e calcular o volume de ambos os ovários, avaliando as massas anexiais, e os algoritmos que medem na população feminina assintomática como os níveis séricos ou as alterações de intervalo do antígeno de câncer, chamado de CA-125. Entretanto, existem alguns problemas em ambos os métodos para a detecção do câncer precocemente. O uso de Doppler também é pensado para uso no rastreamento dessa patologia (BUHLING KJ, et al., 2016; MORERA AC, et al., 2020; KAMAL R, et al., 2018).

\section{Ultrassom Transvaginal}

A ultrassonografia está entre os principais estudos para diagnosticar o câncer ovariano, podendo ser Ultrassom Transabdominal (UTA) e Ultrassom Transvaginal (UTV), porém observa-se que a UTV se destaca ao avaliar e caracterizar as massas anexiais. Na prática, estas estruturas podem ser classificadas em massas císticas simples, anexiais sólidas ou anexiais complexas (MORERA AC, et al., 2020).

As massas císticas simples geralmente são achados benignos e as anexiais sólidas podem ser benignas e malignas, os quais incluem os teratomas císticos, fibromas, ecomas e câncer de ovário. As massas anexiais complexas, por sua vez, possuem características de neoplasias com estruturas internas como nódulos intramurais ou septos (MORERA AC, et al., 2020).

Para o diagnóstico de malignidade com o uso da ultrassonografia, devem ser considerados os seguintes achados: uma massa sólida irregular, uma massa cística multiloculada irregular, ascite, nódulos peritoneais ou outras evidências de metástase e componente sólido ou projeções papilares dentro da parede do cisto, sendo este último o indicador mais sugestivo de malignidade (MORERA AC, et al., 2020).

A fim de distinguir os processos benignos dos malignos, em 2008 foram definidas regras simples para a análise de cistos ovarianos para homogeneizar o diagnóstico ultrassonográfico, que, anteriormente, demandava conhecimento especializado para ser realizado. Nesse estudo foram relatadas boa sensibilidade $(93 \%)$ e boa especificidade $(73 \%)$ e foram definidos critérios benignos e malignos para análise dos achados da ultrassonografia (BÄUMLER M, et al., 2019; MORERA AC, et al., 2020; FROYMAN W, et al., 2017).

Os critérios de benignidade IOTA incluem: cisto unilocular, presença de componentes sólidos e maior diâmetro inferior a $7 \mathrm{~mm}$, cone de sombra, massa multilocular lisa com diâmetro máximo inferior a $10 \mathrm{~cm}$ $(500 \mathrm{~mL})$ e falta de fluxo no Doopler colorido. Os critérios de malignidade, por outro lado, são: tumor sólido irregular, presença de derrame peritoneal, presença de pelo menos quatro vegetações, tumor sólido multiseptado irregular, em que um dos diâmetros é maior que $10 \mathrm{~cm}(500 \mathrm{~mL})$, e pontuação colimétrica forte (BÄUMLER M, et al., 2019).

No que tange os problemas relacionados ao uso do UTV, o volume menor do ovário normal pósmenopausa, localizado lateralmente ao fundo do útero, e outros fatores podem dificultar identificação do ovário. Além disso, revisão sistemática de ensaios randomizados controlados sobre o papel da ultrassonografia transvaginal anual para detectar câncer ovariano na população assintomática evidenciou uma baixa precisão do exame na detecção do câncer, e por isso não há recomendação da realização desse teste na população em geral. Somado a isso, a presença de cistos benignos complexos pode impedir a identificação de tumores malignos (CAMPBELL S e GENTRY-MAHARAJ A, 2018; BUHLING KJ, et al., 2016).

As mulheres com resultados positivos no UTV são submetidas à remoção cirúrgica, e não à uma biópsia, o que pode levar à um procedimento invasivo desnecessário que, por sua vez, pode causar complicações, como infecção, hemorragia, dor, hospitalização prolongada, readmissão, complicações anestésicas e morte. Em contrapartida, os procedimentos cirúrgicos podem proporcionar o aumento da sobrevida ao paciente (CAMPBELL S e GENTRY-MAHARAJ A, 2018; SMITH CG, 2017; FROYMAN W, et al., 2017). 
A biópsia diagnóstica não deve ser realizada devido ao risco de aumentar ou romper o tumor maligno, gerando resultados ainda piores, o que poderia levar à necessidade de tratamento adjuvante ou ao risco de recorrência da doença em estágio inicial. Nesse sentido, a cirurgia definitiva é indicada apenas às mulheres com rastreamento positivo para câncer de ovário com médio a alto risco de malignidade. Existem poucos estudos acerca das possíveis complicações cirúrgicas do câncer de ovário, mas um estudo realizado com 40.000 mulheres em Kentucky evidenciou que 19\% das mulheres com câncer de ovário maligno e $8 \%$ das mulheres com patologia benigna submetidas à cirurgia definitiva tiveram complicações decorrentes desses procedimentos. Entre todas as complicações, 93\% foram definidas como menores (BALDWIN LA, et al., 2017).

Se o intervalo entre a deteç̧ão até a cirurgia for prolongado, o tamanho do tumor provavelmente avançará consideravelmente, o que indica que o tempo de cirurgia deve ser limitado e menor que 90 dias após a detecção da patologia maligna. No Programa de Triagem de Câncer de Ovário de Kentucky, o intervalo de tempo até a cirurgia é menor que 30 dias para evitar a evolução de uma doença avançada na cirurgia. Por outro lado, o ensaio de triagem de câncer de próstata, pulmão, colorretal e ovário. Prostate, Lung, Colorrectal and Ovarian (PLCO) propõe até 9 meses como tempo para a cirurgia, podendo levar ao desenvolvimento de uma doença em estágio avançado (PAVLIK E, 2017).

É necessário o desenvolvimento de novas máquinas de ultrassom com maior resolução de imagem associada à um algoritmo bem padronizado para câncer de ovário nos próximos anos, o que pode fornecer uma melhora em relação à detecção desta patologia (BUHLING KJ, et al., 2016).

\section{CA-125}

O CA-125 é uma molécula com alto peso molecular produzida pelas células mesoteliais do pericárdio, peritônio e da pleura. Além disso, o CA-125 é um antígeno da família das glicoproteínas mucinas, produzido pelo gene MUC16, que normalmente não está presente em ovários de adultos normais, mas na superfície do carcinoma ovariano. O componente extracelular desta glicoproteína é clivado e eliminado pelas células cancerígenas do ovário e, assim, há a possibilidade de sua detecção em fluidos corporais. Essa molécula está presente em neoplasias como as de ovário, endométrio, trompas e mama, e por isso é um dos marcadores tumorais mais utilizados em oncologia ginecológica (HERMIDA LI, et al., 2016; CHARKHCHI P, et al., 2020; DOCHEZ V, et al., 2019).

Nesse contexto, a análise sérica dos níveis da glicoproteína CA-125 é o método bioquímico mais utilizado e mais promissor para a detecção do câncer de ovário e o mais promissor. Este Possui o papel mais significativo na triagem, detecção e controle do câncer de ovário nas últimas quatro décadas. Em pacientes com câncer de ovário, os níveis de CA125 estão positivamente relacionados com a carga tumoral (CHARKHCKI P, et al., 2020).

Aproximadamente $50 \%$ das mulheres com câncer de ovário em estágio inicial e $80 \%$ das mulheres com doença avançada apresentam valores de CA-125 altos. Apesar disso, a especificidade desse método é limitada, levando à altas taxas de falsos positivos, já que esse antígeno também está elevado em uma ampla variedade de condições benignas e malignas, como, por exemplo, endometriose, cirrose, doença inflamatória pélvica e cânceres em outros locais (CAMPBELL S e GENTRY-MAHARAJ A, 2018; MORERA AC, et al., 2020; CHARKHCKI P, et al., 2020).

Alguns estudos realizados observaram que, em condições malignas, o nível de CA-125 tendia a aumentar e, em pacientes sem o câncer de ovário, o nível desse antígeno permanecia estável ou diminuía com o tempo. Tal fato permitiu o desenvolvimento de um algoritmo de risco de câncer de ovário (do inglês, $\mathrm{ROC}$ ), que permite a classificação em baixo, intermediário ou alto risco para desenvolvimento dessa patologia com base no aumento de CA-125. Dessa maneira, para a classificação da neoplasia não se observa apenas o valor absoluto desse antígeno, mas sim se há aumento do nível durante determinado período de tempo. Nesse sentido, alguns estudos propõem a avaliação do algoritmo ROC associado ao UTV como teste secundário para visualização dos ovários caso o teste do CA-125 seja positivo, para melhorar a especificidade dessa técnica (CAMPBELL $S$ e GENTRY-MAHARAJ A, 2018; MORERA AC, et al., 2020). 


\section{Doppler}

O ultrassom do tipo Doppler é capaz de identificar a presença de fluxo de cor dentro do componente papilar ou sólido de um tumor ovariano, o que representa um alto valor preditivo positivo para o diagnóstico diferencial de doenças malignas. Além disso, o uso do Doppler colorido associado à análise espectral de Doppler pulsado amplifica a capacidade diagnóstica do método, uma vez que melhora a caracterização de massas ovarianas mediante medidas quantitativas de fluxo sanguíneo obtidas de vasos tumorais. Considerando que $92 \%$ dos tumores malignos apresentam fluxo sanguíneo, a ausência de fluxo sanguíneo sugere benignidade do tumor. Nesse sentido, a sensibilidade e especificidade desse ultrassom como método diagnóstico de câncer do ovário mostra-se razoável. Porém, ele não é capaz de identificar pequenas lesões ovarianas. (NEBGEN DR, et al., 2020; MORERA AC, et al., 2020; SEHGAL N, 2019).

Além disso, é necessário correlacionar os achados do Doppler com as características morfológicas da lesão, pois o uso isolado de doppler colorido tem pouca utilidade. Um estudo realizado no Reino Unido, por exemplo, apontou que a sensibilidade do UTV para detectar doenças invasivas em todos os estágios é de cerca de $75 \%$ e que, em combinação com o Doppler, o teste apresenta melhor sensibilidade (MORERA AC, et al., 2020).

\section{Reflexão sobre benefícios e danos ocasionados pela prática do rastreamento de câncer de ovário}

A reunião de evidências dos dois maiores estudos nos Estados Unidos e no Reino Unido sobre o rastreamento de câncer de ovário entre mulheres assintomáticas de médio risco não indica que reduza a mortalidade por esse tipo de câncer. Ambos os estudos evidenciaram o aumento dos procedimentos e consequentes complicações desnecessárias decorrentes de exames positivos não resolutivos ou que apresentavam um diagnóstico errôneo. Estima-se que 3\% das mulheres sem câncer que se submeteram à cirurgia diagnóstica tiveram complicação importante. Além disso, tais exames eram caracterizados pela não diminuição da mortalidade da população rastreada em comparação com a população já afetada pelo câncer ovariano. Análises adicionais não encontraram benefícios a longo prazo do rastreio de câncer de ovário (HENDERSON JT, et al., 2017).

Estudos com mulheres com risco baixo ou médio para desenvolvimento de câncer de ovário evidenciaram que o uso de UTV e CA-125 para rastreamento constituiu um maior número de procedimentos e de cirurgias sem diminuir o número de mortes causadas por câncer de ovário, e por esse motivo nenhuma organização médica ou profissional importante recomenda o uso rotineiro de UTV ou do exame de sangue CA-125 para triagem de câncer de ovário em mulheres com risco médio (AMERICAN CANCER SOCIETY, 2020).

Por outro lado, em relação às mulheres com alto risco para câncer de ovário, como as com síndrome genética hereditária, síndrome de Lynch, mutações do gene BRCA ou uma forte história familiar de câncer de mama e ovário, algumas organizações afirmam que o UTV e o CA-125 podem ser oferecidos para triagem. Ainda assim, mesmo nessas mulheres, não foi provado que o uso desses testes para rastreamento diminui o risco de morte por câncer de ovário (AMERICAN CANCER SOCIETY, 2020).

\section{A realização do rastreamento}

A detecção precoce da doença é a melhor alternativa para a redução da mortalidade e aumento da sobrevida. Todavia, embora teoricamente o rastreamento possa melhorar a sobrevida, ainda não há estudos suficientes para validarem o real impacto dessas triagens no prognóstico das pacientes. Nesse sentido, a Força-Tarefa de Serviços Preventivos dos Estados Unidos é contra o rastreamento de câncer ovariano em mulheres assintomáticas, exceto em casos com pré-disposições genéticas. Embora essa doença tenha altas taxas de mortalidade, esta não é muito frequente, o que compromete a viabilidade e a abrangência de um possível programa de triagem (KAMAL R, et al., 2018).

Por fim, apesar dos estudos atuais não demonstrarem benefício nessa triagem, uma publicação mais recente evidenciou uma tendência de menor mortalidade nos pacientes submetidos à triagem após 7-14 anos de acompanhamento (BUHLING KJ, et al., 2016). 


\section{CONSIDERAÇÕES FINAIS}

Os três métodos de rastreamento do câncer de ovário abordados no estudo apresentam pontos positivos e negativos que interferem em sua utilização: a proteína sérica CA-125 é um ótimo marcador tumoral, porém seu teste gera vários resultados falsos positivos; a sensibilidade e especificidade do doppler são razoáveis, mas este não é capaz de detectar pequenas lesões, o que é essencial para o diagnóstico no estágio inicial; e, por fim, o ultrassom transvaginal tem um baixo custo, não é invasivo e é amplamente aceito, mas não consegue identificar tumores na camada epitelial do útero. Portanto, ainda é necessária a realização de mais estudos para encontrar um exame que atenda à todos os critérios de um método de rastreamento, para redução da mortalidade na população feminina por câncer de ovário.

\section{AGRADECIMENTOS E FINANCIAMENTO}

Gostaríamos de agradecer ao Diretório Acadêmico Horizontal - Medicina PUC Minas (DAHMP), que fomentou a escrita científica dentro da Liga Acadêmica de Oncologia da Pontifícia Universidade Católica de Minas Gerais (LAONCO), da qual fazemos parte, e financiou a publicação deste artigo.

\section{REFERÊNCIAS}

1. AMERICAN CANCER SOCIETY. Ovarian Cancer Causes, Risk Factors, and Prevention. Atlanta. American Cancer Society. 2020. Disponível em https://www.cancer.org/cancer/ovarian-cancer/causes-risks-prevention.html. Acesso em: 11 jun. 2021.

2. BALDWIN LA, et al. Complications from Surgeries Related to Ovarian Cancer Screening. Rev. Diagnostics, Lexington, 2017; 7(1): 01-11.

3. BÄUMLER M, et al. Ultrasound screening of ovarian cancer. Hormone Molecular Biology and Clinical Investigation, Berlim, 2019; $41(3):$ 01-06.

4. BUHLING KJ, et al. The role of transvaginal ultrasonography for detecting ovarian cancer in an asymptomatic screening population: a systematic review. Rev. Arch. Gynecol. Obstet., Hamburg, 2017; 295(5): 1259-1268.

5. CAMPBELL S, GENTRY-MAHARAJ A. The role of transvaginal ultrasound in screening for ovarian cancer. Climacteric, [s.I.], 2018; 21(3): 221-226.

6. CHARKHCHI P, et al. CA125 and Ovarian Cancer: A Comprehensive Review. Cancers (Basel). 2020; 12(12):01-29.

7. DANGELO J, FATTINI C. Anatomia Humana Sistêmica e Segmentar. 3rd ed. São Paulo: Atheneu, 2007, 780p.

8. DOCHEZ V, et al. Biomarkers and algorithms for diagnosis of ovarian cancer: CA125, HE4, RMI and ROMA, a review. J Ovarian Res. 2019; 27;12(1): 01-09.

9. FORSTNER R. Early detection of ovarian cancer. European Radiology, [s.I.], 2020; 30(10): 5370-5373.

10. FROYMAN W, et al. Validation of the Performance of International Ovarian Tumor Analysis (IOTA) Methods in the Diagnosis of Early Stage Ovarian Cancer in a Non-Screening Population. Diagnostics, [s.I.], 2017; 7(2): 01-11.

11. HENDERSON JT, et al. Screening for Ovarian Cancer: Updated Evidence Report and Systematic Review for the US Preventive Services Task Force. JAMA, [s.I.], 2018; 319(6): 595-606.

12. HERMIDA LI, et al . Marcadores Tumorales. Rev Clin Med Fam, Albacete, 2016; 9(1): 31-42.

13. INSTITUTO NACIONAL DE CÂNCER. Tipos de câncer: Câncer de Ovário - versão para Profissionais de Saúde. Rio de Janeiro: INCA; 2021. Disponível em: https://www.inca.gov.br/tipos-de-cancer/cancer-de-ovario/profissional-desaude. Acesso em: 11 jun. 2021.

14. KAMAL R, et al. Ovarian cancer screening-ultrasound; impact on ovarian cancer mortality. The British Journal of Radiology, [s.I.], 2018; 91(1090): 01-08.

15. KOSHIYAMA M, et al. Subtypes of ovarian cancer and ovarian cancer screening. Rev. Diagnostics, Kyoto, 2017; 7(12): 01-10.

16. MORERA AC, et al. Cáncer de Ovario: Tamizaje y diagnóstico imagenológico. Med. leg. Costa Rica, Heredia, 2020; 37(1): 54-61.

17. NEBGEN DR, et al. Novel Approaches to Ovarian Cancer Screening. Current Oncology Reports, Texas, 2020, 21(8): $01-17$.

18. PAVLIK E. Ten Important Considerations for Ovarian Cancer Screening. Diagnostics, [s.I.], 2017; 7(2): 1-13

19. SEHGAL N. Eficácia da ultrassonografia Doppler em cores na diferenciação de massas ovarianas. J Midlife Health, [s.I.], 2019; 10(1): 22-28.

20. SMITH CG. A Resident's Perspective of Ovarian Cancer. Rev. Diagnostics, Lexington, 2017; 7(2): 01-17. 